\title{
Down-Regulation of miR-141 Induced by Helicobacter Pylori Promotes the Invasion of Gastric Cancer by Targeting STAT4
}

\author{
Xiaoying Zhou ${ }^{a, b, d}$ Yang Xia ${ }^{b, c, d}$ Jing Sula, Guoxin Zhang ${ }^{a, b}$ \\ aDepartment of Gastroenterology, First Affiliated Hospital of Nanjing Medical University, ${ }^{\text {bFirst Clinical }}$ \\ Medical College of Nanjing Medical University, 'Department of thoracic and cardiovascular surgery, \\ First Affiliated Hospital of Nanjing Medical University, Nanjing, China; ${ }^{\mathrm{d} T h e s e}$ authors contributed \\ equally to the work
}

\section{Key Words}

MicroRNA-141 • STAT4 • Invasion • Gastric cancer

\begin{abstract}
Background: The association between Helicobacter pylori infection and gastric cancer has been identified recently. However, the molecular mechanism remained largely unknown. Methods and Results: We found that miR-141 was decreased in Helicobacter pylori positive specimens $(n=75)$ compared with negative tissues $(n=75)$. The knockdown of miR-141 enhanced the invasion ability of gastric cancer cells; meanwhile, over-expression of miR-141 could inhibit the abilities of gastric cancer cells in vitro. A luciferase assay revealed that miR-141 was directly bound to the 3'-untranslated regions (3'-UTR) of STAT4. STAT4 was found up-regulated at mRNA and protein levels, as shown by qRT-PCR and western blot. Over-expression of STAT4 was used to mimic miR-141 action in the invasion of gastric cancer. Conclusion: MiR-141 may play a pivotal role in controlling gastric cancer invasion through regulating STAT4 and maybe a potential target to treat gastric cancer.
\end{abstract}

Copyright $@ 2014$ S. Karger AG, Basel

\section{Introduction}

Helicobacter pylori (H. pylori) is a spiral-shaped gram-negative microorganism with a flagellum. Infection with H. pylori is the strongest risk factor for the development of gastric carcinoma, which is the second most common cause of cancer-related death worldwide [1]. 
WHO has defined H. pylori as type I pathogen of gastric cancer [2]. This results from the consequences of long-term interactions between the bacterium and its human host. Despite the great progress that has been made recent decades about the relationship between $\mathrm{H}$. pylori infection and gastric cancer, the molecular mechanism of them has not been fully recognized [3].

MiRNAs are a class of small, non-coding RNAs that play important roles in various biological processes [4]. Bioinformatic methods predicted that in approximately one-third of all mammalian genes, especially those in down-stream of various signal transduction pathway, were targeted by miRNAs [5]. Interestingly, accumulating evidence has implicated roles of miRNAs in human cancer. While many oncogenic miRNAs, including miR-184, miR95 and miR-27a, are over-expressed in tumors, while some tumor-suppressive miRNAs are down-regulated [6]. High-throughput techniques have been used to screen miRNAs differentially expressed between human nonmalignant and malignant samples, and a number of miRNAs deregulated in numerous human tumors were found, including lung, breast, liver, esophageal and prostate cancers [7]. Among these miRNAs, miR-141, a member of the miR-200 family, is over-expressed in ovarian and colorectal cancers and down-regulated in prostate, hepatocellular, and renal cell carcinoma, raising a controversial issue about the role of miR-141 in cancer progression [8].

Signal transducer and activator of transcription 4 (STAT4) is widely up-regulated in many carcinomas and its expression correlates with the clinicopathological characteristics (malignant properties) of human cancers [9]. A previous study demonstrated that miR-155 regulates cell functions by targeting the 3'-UTR of STAT4 [10]. In this study, we investigated the function of miR-141 in gastric carcinogenesis. In gain-of-function and loss-of-function studies, we found that miR-141 inhibits gastric cancer cell migration and invasion. By in silico prediction and experimental validation, we identified STAT4 as the functional downstream targets of miR-141, and showed that they play distinct roles in regulating gastric cancer development.

\section{Materials and Methods}

\section{Clinical Samples}

150 patients with or without H. pylori infection had undergone gastroscope at The First Affiliated Hospital of Nanjing Medical University, Jiangsu Province, China from March 2013 and July 2013. The samples taken from the 150 patients were collected, immediately snap frozen in liquid nitrogen, and stored at -80 ${ }^{\circ} \mathrm{C}$ until RNA extraction. The samples were identified as positive when rapid urease test was positive. These selected patients were also confirmed by ${ }^{13} \mathrm{C}$ breath test. This study was approved by the Ethical Committee of the first affiliated hospital of Nanjing Medical University, and every patient had written informed consent.

Cell culture

SGC-7901 gastric cancer cell lines (purchased from ATCC) were employed for the present study and were cultured in RPMI-1640 medium supplemented with $10 \%$ fetal bovine serum (Gibico) and penicillin $(100 \mathrm{U} / \mathrm{ml})$. Cells were cultured at $37^{\circ} \mathrm{C}$ in a humidified atmosphere with $5 \% \mathrm{CO}_{2}$.

\section{Isolation of total RNA and Quantitative RT-PCR}

Total RNA was extracted from collected tissues using TRIzol (Tarkara, Japan) and then both miRNA and mRNA were reverse transcribed to cDNA. The reverse transcription was performed by using reverse transcription kit (Takara, Otsu, Shiga, Japan). The expression of mature miRNAs and potential target genes were measured by qRT-PCR with SYBR Green PCR Kit (Takara) on Applied Biosystems StepOne-Plus RealTime PCR System and human U6 RNA was amplified as an internal control. The relative expression ratio of miR-141 in each paired positive and negative tissue was calculated by the $2^{-\Delta \Delta C T}$ method. PCR reactions were performed with the following primers: for hsa-miR-141, forward, 5'-CAUCUUCCAGUACAGUGUUGGA-3' and for U6, forward, 5'-CTCGCTTCGGCAGCACA-3'. Relative expression levels of STAT4 mRNA were examined by SYBR Green real-time PCR (RT-PCR) and normalized to GAPDH. The primers for STAT4 were forward, 
5' AGCCATCTCGGAGGAATA 3' and reverse, 5' CAGACAACCGGCCTTTAT 3'; and for GAPDH, forward, 5'-CGTGGGCCGCCCTAGGCACCA-3' and reverse, 5'-TTGGCTTAGGGTTCAGGGGGG-3'. RT-PCR was performed by using the ABI 7500 Fast Real-Time PCR system (ABI, CA, USA).

\section{Immunohistochemistry}

The positive and negative tissues were fixed in $4 \%$ paraformaldehyde and cut from paraffin block to $5 \mu \mathrm{m}$ thickness. After dewaxing with xylene and rehydration with a graded series of ethanol, slides were heated in the autoclave for three minutes using citrate sodium buffer (PH 6.0) and incubated with the antibody STAT4 (polyclonal, 1:1000 dilution, Santa CruZ) at $4{ }^{\circ} \mathrm{C}$ overnight. Blocking serum or antibody dilution buffer was used as negative controls. The antibodies utilized before were the same as for Western blot analysis. Photographs were taken by microsope (Nikon, ECLIPSE 50i) and software NIS-Elements v4.0. The number of positive staining cells showing immunoreactivity of STAT4 in ten representative microscopic fields was counted, and the percentage of positive cells was also calculated. The percentage scoring of immunoreactive tumor cells was described as follows: 0 (0 \%), 1 (1-10\%), 2 (11-50\%), and 3 (>50 \%). The intensity was scored as follows: 0 (negative), 1 (weak), 2 (moderate), and 3 (strong). The expression level of STAT4 was measured by multiplying the percentage and the intensity score. High expression samples means tumors with a multiplied score exceeding 4 while the others were considered to be low expression.

\section{Wound-healing assay}

A wound-healing assay was used to assess capacity for tumor cell motility. Briefly, cells $\left(1^{*} 10^{6} /\right.$ well $)$ were seeded in six-well plates, cultured overnight, and transfected with miR-141 mimics or inhibitors and the respective controls. On reaching confluence, the cell layer was scratched with a sterile plastic tip and then washed with culture medium twice and cultured again for up to 48 or $72 \mathrm{~h}$ with serum-reduced medium containing 1\% FBS. Photo images of the plates were taken under a microscope. The gap closure was measured at 48 or $72 \mathrm{~h}$ and the data were summarized based on sextuple assays for each experiment.

\section{Cell migration and invasion assay}

Cell motility was measured using an 8- $\mu$ m-pore polycarbonate membrane Boyden chamber insert in a Transwell apparatus (Millipore, MA, USA). The transfected cells (miR-141 mimics, miR-141-inhibitors and NC) growing in the log phase was treated with trypsin/EDTA solution, washed once with serum-containing RPMI-1640 medium. A total of $1 \times 10^{5}$ cells in $0.2 \mathrm{ml}$ serum-free RPMI-1640 medium were seeded on a Transwell apparatus. RPMI-1640 containing 10\% FBS $(600 \mu \mathrm{l})$ was added to the lower chamber. An invasion assay was conducted following the same procedure, with the exception that the filters of the transwell chambers were coated with $45 \mu \mathrm{g}$ Matrigel (BD Biosciences; San Jose, CA, USA). Following incubation of the cells for $24 \mathrm{~h}$ at $37^{\circ} \mathrm{C}$ in a $5 \% \mathrm{CO}_{2}$ incubator, cells on the top surface of the insert were removed by wiping with a cotton swab. The cells that invaded to the bottom surface of the insert were fixed in the $100 \%$ precooling methanol for $10 \mathrm{~min}$, stained in $0.5 \%$ crystal violet for $30 \mathrm{~min}$, then rinsed in PBS and subjected to microscopic inspection. The values for invasion were obtained by counting three fields per membrane and represented the average of three independent experiments.

\section{Bioinformatics analysis}

In this study we used bioinformatics method to predict the potential targeting gene of miR-141. As the results in the starBase (http://starbase.sysu.edu.cn/) database, STAT4 was the candidate gene we picked up. The bioinformatic software showed that 3'-UTR of STAT4 binds to miR-141 with the high score.

\section{Western blotting}

Total proteins were prepared from the established cells, quantities using a protein assay (BCA method, Thermo, USA). The STAT4 protein was analyzed by western blot analysis using STAT4 Rabbit Monoclonal Antibody and GAPDH was used as an internal control. Proteins were fractionated by sodium dodecyl sulfate polyacrylamide gel electrophoresis (SDS-PAGE) transferred to polyvinylidene fluoride (PVDF) membrane, blocked in 5\% dry milk at room temperature for 1 hour and immunostained with antibodies at $4^{\circ} \mathrm{C}$ overnight using anti-STAT4 (1:1000, CST, USA) and anti-GAPDH (1:5000, sigma, USA). All results were visualized through a chemiluminescent detection system (Pierce ECL Substrate Western blot detection system, Thermo, Pittsburgh, PA) and then exposed in Molecular Imager ChemiDoc XRS System (Bio-Rad, Hercules, CA). 
Fig. 1. MiR-141 is down-regulated in H. Pylori positive patients. (A) The expression levels of miR-141 in human H. Pylori positive tissues and normal tissues relative to U6 were determined by qRT-PCR ( $\mathrm{n}=75, \mathrm{p}<0.0067)$. (B) The expression levels of miR-141 in CG and CAG in $\mathrm{H}$. Pylori positive tissues $(\mathrm{p}<0.05)$. Data are represented as mean \pm SD. * indicates $\mathrm{P}<0.05$.

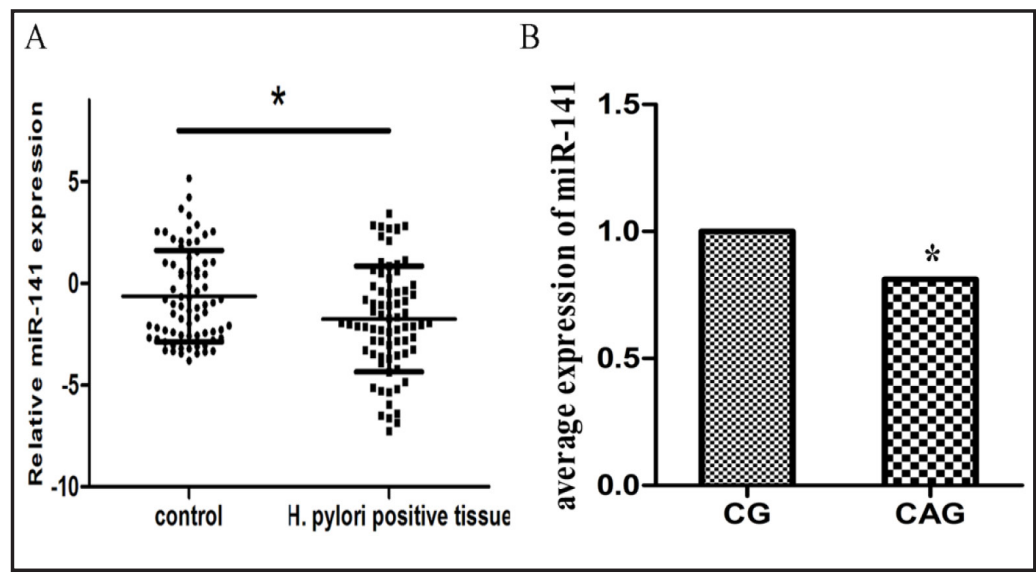

Plasmid construction and cell transduction

3'-UTR sequence of STAT4 which was predicted to interact with miR-141 or a mutant sequence with the predicted target sites were inserted into the KpnI and SacI sites of pGL3 promoter vector (Invitrogen). They were named pGL3-STAT4 and pGL3-STAT4-mut. The cells were plated onto 6-well plates and were transfected with $100 \mathrm{ng}$ of pGL3-STAT4 or pGL3-STAT4-mut, and miR-141 mimics (50nM) by using Lipofectamine 2000 (Invitrogen Corp, CA, USA). We normalized the differences in transfection efficiency by co-transfecting a Renilla luciferase vector pRL-SV40 (5 ng).

STAT4 gene was synthesized (purchased from Genscript, Piscataway, NJ) with restrictive digestion using Mlu I and subcloned pLV-GFP plasmid, and named pLV-GFP-STAT4. Recombinant lentivirus was generated from 293T cells using calcium phosphate precipitation. SGC-7901 cell lines were transfected with lentivirus using polybrene $(8 \mathrm{ug} / \mathrm{ml})$.

\section{Luciferase activities analysis}

The SGC-7901 cells were cultured in 24-well plates and transfected with $0.2 \mu \mathrm{g}$ of either wide-type or mutant pGL/STAT4 plasmid containing firefly luciferase, together with $0.01 \mu \mathrm{g}$ of the pGL-STAT4 vector (Invitrogen) containing renilla luciferase and $1 \mu \mathrm{g}$ oligonucleotides. Transfection was performed using Lipofectamine 2000 reagent (Invitrogen). Relative luciferase activity was calculated 48h post-transfection by the Dual Luciferase Reporter Assay (Promega).

\section{Statistical methods}

Differences between groups were compared using Student's t-test for continuous variables. The relations of miR-141 with STAT4 mRNA or protein were analyzed by correlation coefficients and linear regression analysis. $P$ values $<0.05$ was considered as statistical significance.

\section{Results}

MiR-141 is aberrantly down-regulated in H. Pylori positive tissue

First, we analyzed the expression levels of miR-141 in both H. Pylori positive tissues and normal tissues by qRT-PCR. Significantly, we found that miR-141 expression was decreased in $\mathrm{H}$. Pylori positive tissues $(\mathrm{n}=75, \mathrm{p}<0.0067$; Fig. 1A). Among the $\mathrm{H}$. pylori positive tissues, we found that CAG had a lower average expression of miR-141 than CG ( $p<0.05$; Fig. 1B). The aberrant expression level of miR-141 suggested that miR-141 may play a potential role in H. Pylori induced gastric cancer progression and development. Therefore, based on this expression pattern, we chose the SGC-7901 cell lines to determine the functions of miR-141 in gastric cancer. 
Fig. 2. Effects of miR-141 expression on SGC-7901 cells invasion. (A) MiR-141 level in SGC-7901 cells transfected with miR-141 mimics, miR-141 inhibitor, negative control (NC) and inhibitor negative control (iNC). The result was validated by real-time PCR. (B) The images photographed at $0 \mathrm{~h}$ (upper) and 24 $\mathrm{h}$ (lower) postwounding were shown at magnification of $\mathrm{x} 200$. C. Transwell assay was performed as described in Materials and Methods. Cells were treated with miR-141 mimics, miR-141 inhibitor, NC and iNC for $24 \mathrm{~h}$. The representative images of

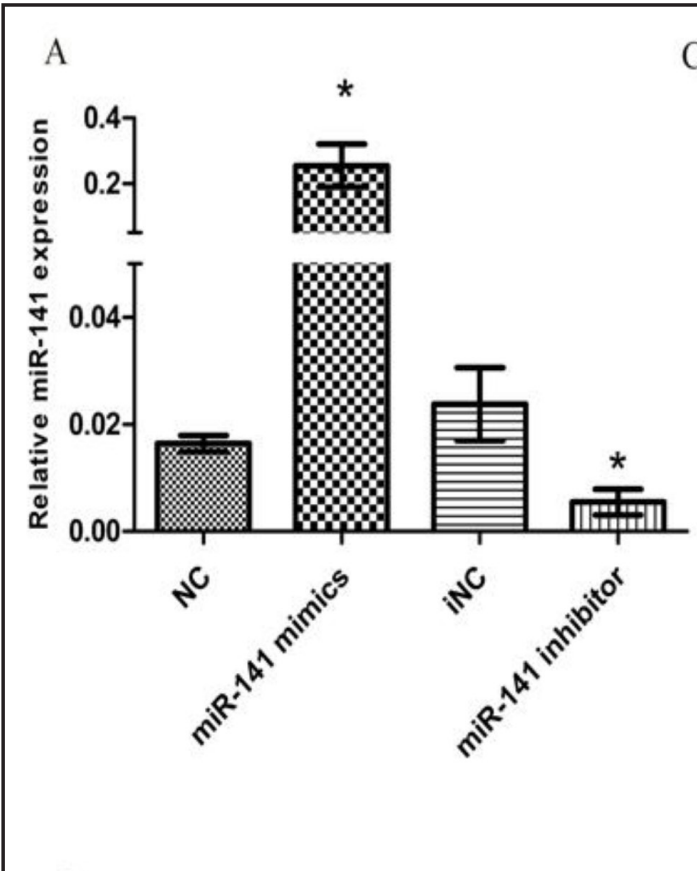

B

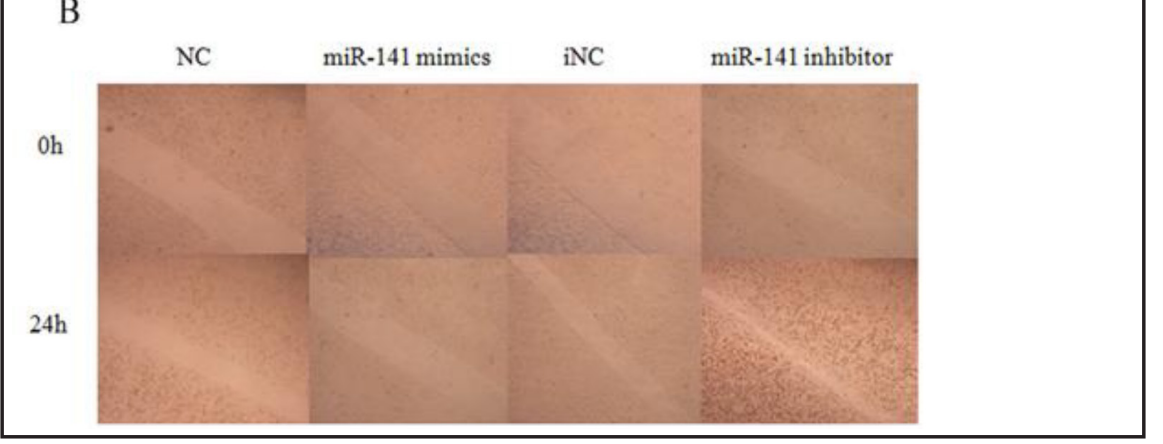
invasive cells at the bottom of the membrane stained with crystal violet were visualized as shown. The quantifications of cell migration were presented as percentage migrated cell numbers. All experiments were performed in triplicate and presented as mean \pm SD. * indicates significant difference compared with control group $(\mathrm{P}<0.05)$. Every independent experiment was performed 3 times.

Ectopic miR-141 inhibits the migration and invasion of SGC-7901 cells in vitro

The low expression of miR-141 in H. Pylori positive tissues prompted us to examine the role of miR-141 in the invasion of gastric cancer. SGC-7901 cells were transfected with miR-141 mimics and miR-141 inhibitors. The transfection efficiency was then validated by qRT-PCR (Fig. 2A). Using wound healing assay, we discovered that the over-expression of miR-141 could suppress SGC-7901 cell invasion, while its knockdown induced cell invasion (Fig. 2B). Consistently, Matrigel invasion assay showed that over-expression of miR-141 attenuated SGC-7901 cell invasion, whereas the knockdown of miR-141 could reverse the affection (Fig. 2C). We also conducted the invasion and scratch wound assays on another gastric cancer cell line, MKN45 with similar results (data not shown). The results suggested that miR-141 could inhibit invasion of the gastric cancer cell line in vitro.

MiR-141 up-regulated STAT4 expression in gastric cancer

We identified the downstream targets of miR-141 by computational prediction to elucidate the mechanisms by which miR-141 suppressed gastric cancer cell invasion. Among hundreds of genes predicted by online miRNA target prediction website (starBase, http:// 


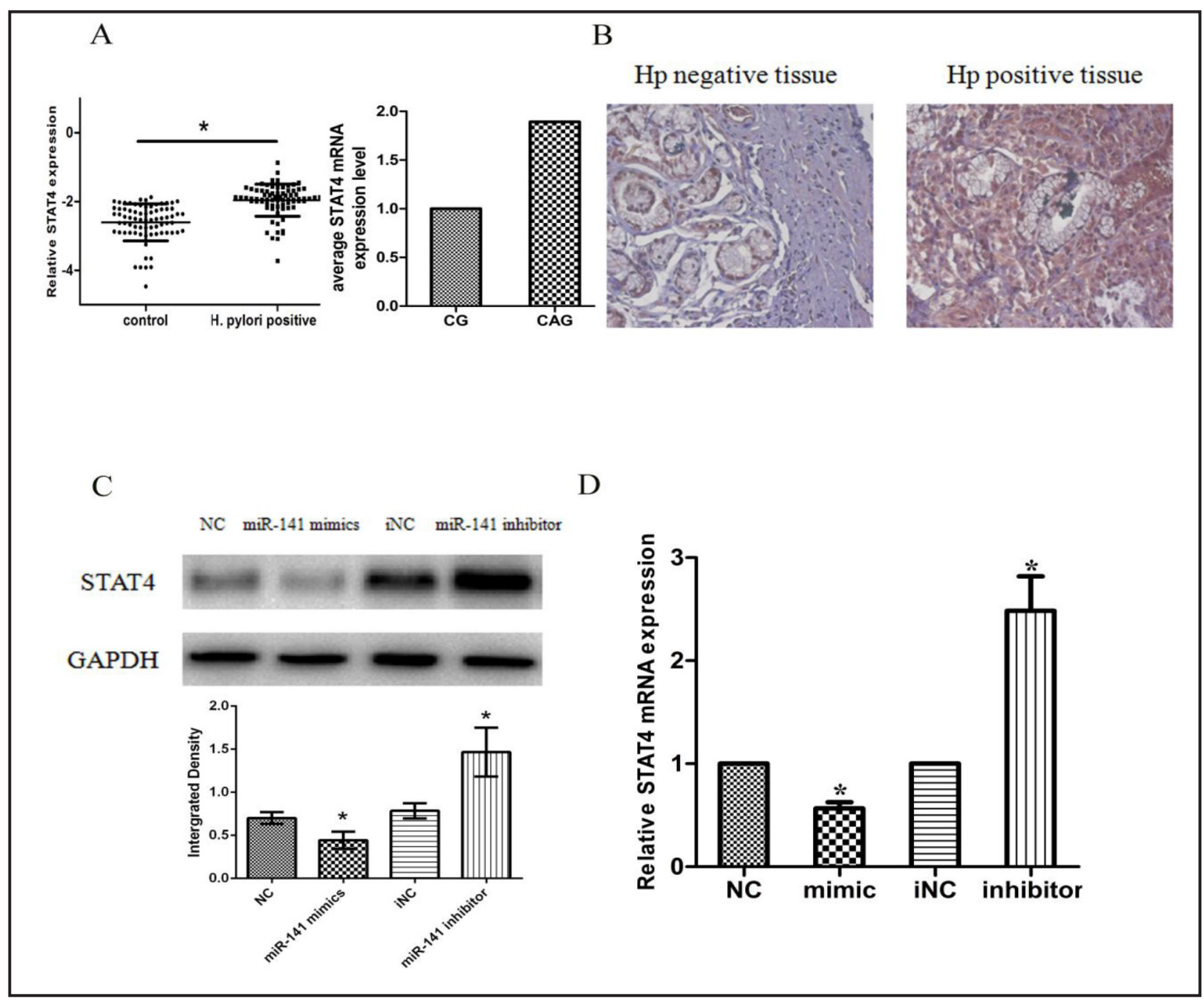

Fig. 3. Up-regulation of STAT4 in H. pylori positive tissues. (A) The mRNA levels of STAT4 relative to GAPDH in human H. Pylori positive tissues including CG and CAG and normal tissues were evaluated by qRT-PCR. Data were presented as scattergram of the median. * Significantly different compared with that of control $(\mathrm{P}<0.05)$. (B) STAT4 protein expression in H. Pylori positive and negative tissues was detected by Immunohistochemical staining. (C) STAT4 protein levels in SGC-7901 cells transfected with negative control (NC), miR-141 mimics; miR-141 inhibitor and iNC were analyzed through Western-blotting. GAPDH was used as a loading control. Average values of integrated optical density (IOD) were assessed by analyzing five fields per slide and recorded in the histogram. (D) STAT4 mRNA levels in SGC-7901 cells transfected with NC, miR-141 mimics; miR-141 inhibitor and iNC were analyzed through qRT-PCR. The relative expression of STAT4 was calculated by the $2^{-\Delta \Delta C T}$ method. Data are represented as mean \pm SD. * indicates $\mathrm{P}<0.05$.

Table 1. Expression level of STAT4 in Helicobacter pylori positive tissues. CG: chronic gastritis; CAG: chronic atrophic gastritis; * indicates $\mathrm{P}<0.05$

\begin{tabular}{|c|c|c|c|c|}
\hline \multirow[t]{2}{*}{ Factor } & \multirow[t]{2}{*}{ Sample N=75 } & \multicolumn{2}{|c|}{$\begin{array}{c}\text { STAT4 expression } \\
\text { (immnohistochemistry) }\end{array}$} & \multirow[t]{2}{*}{ P value } \\
\hline & & Low & High & \\
\hline Gender & & & & 0.773 \\
\hline Male & 42 & 15 & 27 & \\
\hline Female & 33 & 11 & 22 & \\
\hline Age(years) & & & & 0.521 \\
\hline$<40$ & 34 & 14 & 20 & \\
\hline$\geq 40$ & 41 & 12 & 29 & \\
\hline Histology & & & & $0.005^{*}$ \\
\hline CG & 33 & 11 & 22 & \\
\hline $\mathrm{CAG}$ & 41 & 14 & 27 & \\
\hline
\end{tabular}

starbase.sysu.edu.cn/), we forcused our attention on STAT4. STAT4 had been reported to regulate gastric cancer progression or act as an underlying functional target of miR-141. To identify the prediction, through qRT-PCR and immunohistochemistry, we found that STAT4 
Fig. 4. Effects of miR-141 on STAT4 expression by luciferase reporter assay. The potential miR-141 binding site at the 3UTR of STAT 4 mRNA was computationally predicted by starBase. SGC-7901 cells were co-transfected with miR-141 mimics (or negative control) with pGL3-STAT4 (or pGL3-STAT4mut) vector. Luciferase activity was normalized by the ratio of firefly and Renilla luciferase signals. * indicates $\mathrm{P}<0.05$.
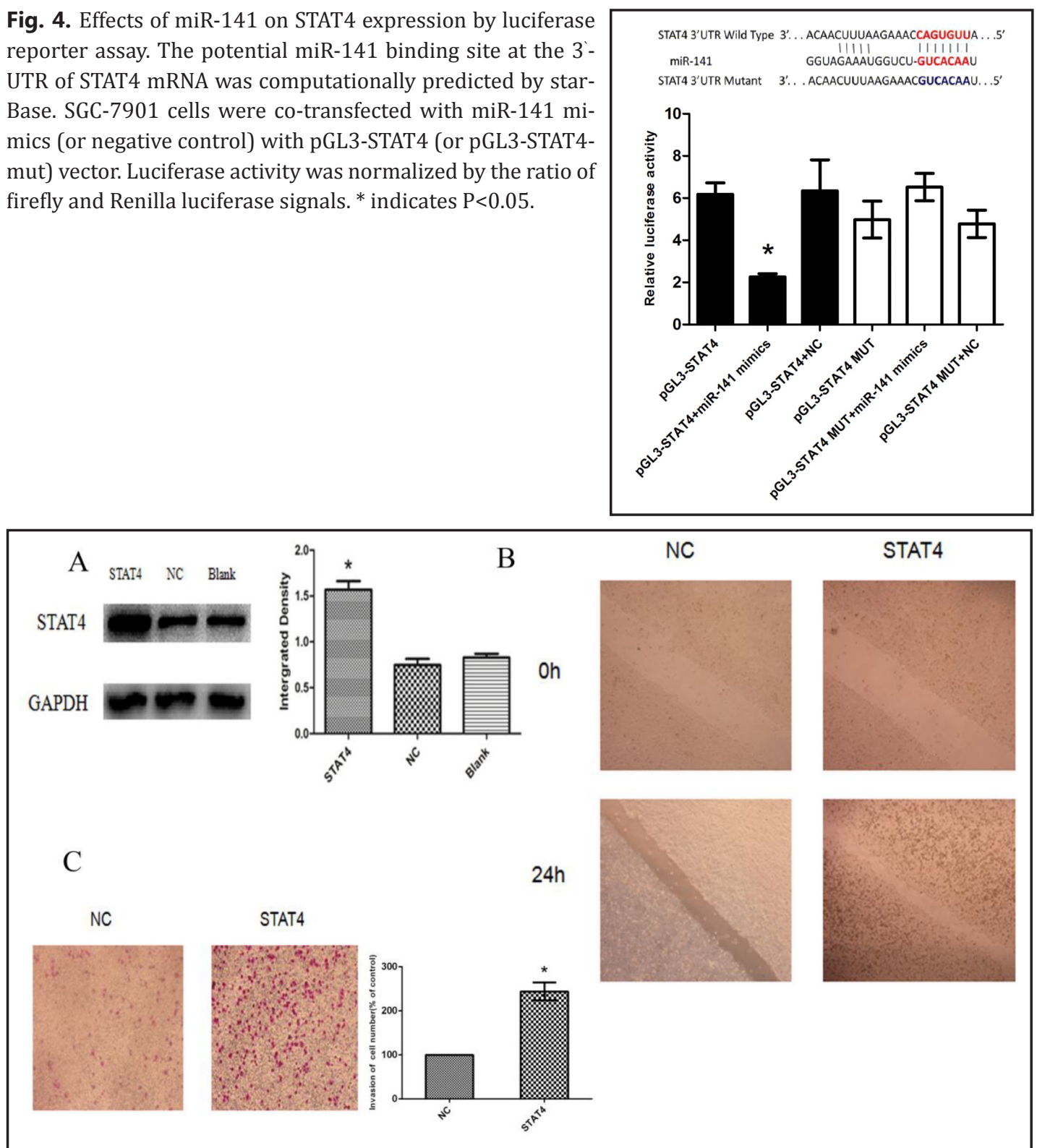

Fig. 5. Effects of over-expression of STAT4 by transfecting with lentivirus on SGC-7901 cell invasion. (A) STAT4 protein expression was analyzed by Western blotting in SGC-7901 cells by transfecting with lentivirus, control plasmid, and blank. GADPH was shown as internal control (left). Average values of integrated density were assessed by analyzing five fields per slide and recorded in the histogram (right). (B) Wound healing assay of these images at $0 \mathrm{~h}$ (upper) and $24 \mathrm{~h}$ (lower) post-wounding were shown at magnification of x200. (C) Transwell assay was performed as described in Materials and Methods. STAT4 over-expressed and NC groups were respectively stained. The representative images of invasive cells at the bottom of the membrane stained with crystal violet were visualized as shown. The quantifications of cell migration were presented as percentage migrated cell numbers. All experiments were performed in triplicate and presented as mean $\pm \mathrm{SD}$. * indicates significant difference compared with control group $(\mathrm{P}<0.05)$. Every independent experiment was performed 3 times.

was up-regulated in H. pylori positive tissues. Its expression was also higher in CAG than CG in H. pylori positive tissues. ( $n=75$, $p<0.0001$; Fig. $3 A$ and B), which was inversely correlated with miR-141. Using immunohistochemistry, we also discovered that STAT4 expression was significantly associated with gastric histology $(\mathrm{p}<0.05$; Table 1$)$. In vitro, we then determined 
the expression of STAT4 in response to the changes in miR-141 expression. Western blot and qRT-PCR assays showed that over-expression of miR-141 could significantly down-regulate the mRNA and protein expression of STAT4, while the effect of knockdown of miR-141 was the opposite (Fig. 3C and D).

\section{STAT4 is a direct target gene of miR-141 in gastric cancer cells}

Though STAT4 has been regarded as a direct target of miR-141, it is unclear whether miR-141 can directly recognize the 3'-UTR of STAT4 mRNA. According to the predicted target site from starBase, we cloned the 3'-UTR fragment containing the predicted site into the pGL3 luciferase reporter vector (pGL3-STAT4). The 3'-UTR fragment with mutated sequence within the predicted target site was also cloned as a control (pGL3-STAT4-mut). The results showed that co-transfection with miR-141 mimics and the pGL3-STAT4 vector decreased luciferase activity in SGC-7901 cells. However, miR-141 mimics did not have the effect on luciferase activity when the cells were transfected with pGL3-STAT4-mut vector (Fig. 4). These data suggested that STAT4 gene was one of the direct targets of miR-141.

Over-expression of STAT4 has the effect of miR-141 on gastric cancer cells invasion

To determine STAT4 as the functional effector of miR-141 in gastric cancer invasion, we over-expressed STAT4 by transfecting with lentivirus and examined its effects in gastric cancer cells. Over-expression of STAT4 in the cell line was confirmed by western blotting (Fig. 5A). Through wound healing and the Matrigel invasion assay, we then discovered that the over-expression of STAT4 significantly promoted SGC-7901 cells invasion (Fig. 5B and 5 C).

\section{Discussion}

Recently, H. pylori infection rate is increasing, especially in developing countries [11]. Clinical evidence suggested that H. pylori is related to gastric cancer, but the mechanism remained unclear [12]. Majority of cancer-related death is caused by metastasis and recent studies have demonstrated that miRNAs play an important role in the regulation of metastasis process [13]. However, their association with gastric cancer remains largely unknown. Very recently, miR-141 has been shown to be up-regulated in bone-metastatic prostate cancer and colon cancer [14]. Previous studies have demonstrated that the expression profiling of miR141 was tissue-specific and that it might have divergent functions depending on the tumor tissue or cell type [15]. It has been reported that miRNA repression of mRNA is dependent on the conditions of specific cellular targets [16]. Here, we present evidence that miR-141 was significantly down-regulated in H. Pylori positive tissues and that over-expression of miR-141 suppressed the growth of cells derived from gastric tumor.

In this study, we investigated the role of miR-141 and identified its targets in Helicobacter pylori infection and gastric cancer. We first quantified the expression of miR-141 in $\mathrm{H}$. Pylori infection tissues and normal tissues and we found that the miR-141 expression is significantly lower in H. Pylori infected patients. We also found that the expression is related to the histology of the patients, that is, the expression of miR-141 is significantly lower in $\mathrm{H}$. Pylori positive patients with chronic atrophic gastritis than chronic non-atrophic gastritis. This expression pattern raises that possibility that miR-141 is related to different kinds of gastritis and the more severe the patients were, the lower the expression of miR-141 was.

The functional study of miR-141 in malignancy was previously reported in renal cell carcinoma. Hirofumi et al postulated that miR-141 was a potential metastasis inhibitor in renal cancer and reported that upregulation of miR-141 inhibited migration of cancer cells. They demonstrated that miR-141 induced dysregulation of genes related to migration and invasion, including ZEB1 [17]. It was indicated that miR-141 was likely to have a number of targets through which it regulated biological functions on cancer cells. 
Based on the miR-141 expression level, we rationally chose SGC-7901 cells for the subsequent gain-of-function and loss-of-function studies, respectively. The results suggest that down-regulation of miR-141 in gastric cancer cell is related to its progression. Although a large number of human miRNAs have been reported, many of their mRNA targets remain unidentified [18]. In this study, we used a combined bioinformatics and experimental approach to identify that STAT4 are the functional downstream target of miR-141.

STAT4 is a transcription factor belonging to the signal transducer and activator of transcription protein family and has been shown to play a role in the acquisition of the invasive and metastatic phenotype in colon and rectal cancers [19]. We show that STAT4 is critically involved in gastric cancer metastasis as knockdown of STAT4 suppresses cell migration, invasion and metastasis.

To conclude, our evidence indicated that miR-141 was down-regulated, while STAT4 was up-regulated in H. Pylori infection and gastric cancer. Ectopic miR-141 expression decreased STAT4 expression at the mRNA and protein levels in the gastric cancer cell line. We also demonstrated that miR-141 was directly bound to the 3'-UTR of STAT4. MiR-141 expression inhibited gastric cancer invasion via repression of STAT4. Furthermore, the miR141-STAT4 pathway that we identified may be exploited in a therapeutic approach for the treatment of H. Pylori related cancers.

\section{Abbreviations}

3'-UTR: 3' (untranslated regions); qRT-PCR (Quantitative Real Time- Polymerase Chain Reaction); NuRD (nucleosome remodeling and histone deacetylation); FBS (fetal calf serum).

\section{Acknowledgements}

We would like to thank the funding of the study provided by National Natural Science Foundation of China (No.81270476 and 81072032) and the Priority Academic Program Development of Jiangsu higher Education Institutions (PAPD).

\section{Disclosure Statement}

The authors declare no conflict of interest.

\section{Reference}

1 Bhandari A, Crowe SE: Helicobacter pylori in gastric malignancies. Curr Gastroenterol Rep 2012;14:489496.

-2 Selgrad M, Bornschein J, Rokkas T, Malfertheiner P: Helicobacter pylori: Gastric cancer and extragastric intestinal malignancies. Helicobacter 2012;17:S30-35.

3 Lamb A, Chen LF: Role of the helicobacter pylori-induced inflammatory response in the development of gastric cancer. J Cell Biochem 2013;114:491-497.

4 Zhang B, Pan X, Cobb GP, Anderson TA: Micrornas as oncogenes and tumor suppressors. Dev Biol 2007;302:1-12.

5 Einat P: Methodologies for high-throughput expression profiling of micrornas. Methods Mol Biol 2006;342:139-157.

6 Zhang Y, Li M, Wang H, Fisher WE, Lin PH, Yao Q, Chen C: Profiling of 95 micrornas in pancreatic cancer cell lines and surgical specimens by real-time pcr analysis. World J Surg 2009;33:698-709. 
Zhou et al.: miR-141 and STAT4

7 Luo Z, Wen G, Wang G, Pu X, Ye S, Xu Q, Wang W, Xiao Q: Microrna-200c and -150 play an important role in endothelial cell differentiation and vasculogenesis by targeting transcription repressor zeb1. Stem Cells 2013;31:1749-1762.

-8 Feng B, Wang R, Chen LB: Review of mir-200b and cancer chemosensitivity. Biomed Pharmacother 2012;66:397-402.

-9 Watson CJ: Stat transcription factors in mammary gland development and tumorigenesis. J Mammary Gland Biol Neoplasia 2001;6:115-127.

10 Ha TY: Micrornas in human diseases: From autoimmune diseases to skin, psychiatric and neurodegenerative diseases. Immune Netw 2011;11:227-244.

-11 Shmuely H, Katicic M, Filipec Kanizaj T, Niv Y: Helicobacter pylori and nonmalignant diseases. Helicobacter 2012;17 Suppl 1:22-25.

12 Miehlke S, Kirsch C, Dragosics B, Gschwantler M, Oberhuber G, Antos D, Dite P, Lauter J, Labenz J, Leodolter A, Malfertheiner P, Neubauer A, Ehninger G, Stolte M, Bayerdorffer E: Helicobacter pylori and gastric cancer:Current status of the austrain czech german gastric cancer prevention trial (prisma study). World J Gastroenterol 2001;7:243-247.

13 Li M, Fu W, Wo L, Shu X, Liu F, Li C: Mir-128 and its target genes in tumorigenesis and metastasis. Exp Cell Res 2013;319:3059-3064.

14 Brase JC, Johannes M, Schlomm T, Falth M, Haese A, Steuber T, Beissbarth T, Kuner R, Sultmann H: Circulating mirnas are correlated with tumor progression in prostate cancer. Int J Cancer 2011;128:608616.

15 Yanagawa B, Lovren F, Pan Y, Garg V, Quan A, Tang G, Singh KK, Shukla PC, Kalra NP, Peterson MD, Verma S: Mirna-141 is a novel regulator of bmp-2-mediated calcification in aortic stenosis. J Thorac Cardiovasc Surg 2012;144:256-262.

16 Liu H, Kohane IS: Tissue and process specific microrna-mrna co-expression in mammalian development and malignancy. PLoS One 2009;4:e5436.

17 Zawislak CL, Beaulieu AM, Loeb GB, Karo J, Canner D, Bezman NA, Lanier LL, Rudensky AY, Sun JC: Stagespecific regulation of natural killer cell homeostasis and response against viral infection by microrna-155. Proc Natl Acad Sci U S A 2013;110:6967-6972.

18 Li Y, Kowdley KV: Micrornas in common human diseases. Genomics Proteomics Bioinformatics 2012;10:246-253.

19 Thieu VT, Yu Q Chang HC, Yeh N, Nguyen ET, Sehra S, Kaplan MH: Signal transducer and activator of transcription 4 is required for the transcription factor t-bet to promote thelper 1 cell-fate determination. Immunity 2008;29:679-690. 